\title{
Psiquiatria, História e Arte
}

\section{0 advento do tratamento psiquiátrico moderno: a terapia convulsiva de László Meduna}

\section{Lessons from an early account of convulsive therapy}

\author{
Brigitta Baran M.D. ${ }^{1}$, István Bitter, M.D., Ph.D., D.Sc. ${ }^{2}$, Gábor S. Ungvári M.D., Ph.D. ${ }^{3}$, \\ Zoltán Nagy M.D., Ph.D., D.Sc. ${ }^{4}$, Gábor Gazdag M.D., Ph.D. ${ }^{5}$ \\ ${ }^{1}$ Departamento de Psiquiatria e Psicoterapia da Faculdade de Medicina, Universidade de Semmelweis. \\ 2 Professor catedrático do Departamento de Psiquiatria e Psicoterapia da Faculdade de Medicina, Universidade de Semme/weis. \\ ${ }^{3}$ Departamento de Psiquiatria da Universidade Chinesa de Hong Kong. \\ ${ }^{4}$ Professor e chefe de Departamento do Instituto Nacional de Psiquiatria e Neurologia. \\ ${ }^{5}$ Serviço de Consultoria Psiquiátrica de Ligação. \\ Recebido: 05/05/2008 - Aceito: 20/05/2008
}

\section{Resumo}

A terapia convulsiva (TC) constitui uma das principais contribuições européias ao tratamento psiquiátrico moderno e à psiquiatria biológica. A TC foi introduzida na psiquiatria em 1934 por László Meduna, neuropsiquiatra húngaro. As publicações subseqüentes sobre o primeiro paciente tratado com TC, Zoltán L (ZL), baseiam-se nos artigos e na autobiografia de Meduna. De acordo com essas referências, após quatro anos de estupor catatônico, ZL recebeu TC induzida por cânfora que resultou em plena remissão dos sintomas esquizofrênicos, culminando com alta da instituição. Este artigo reconstrói a história do caso de ZL a partir da recente recuperação de anotações - algumas escritas pelo próprio Meduna - dos Arquivos do Instituto Nacional de Psiquiatria e Neurologia (Hungria). Essas anotações mostram que ZL recebeu repetidas sessões de TC entre 1934 e 1937, primeiramente induzidas por cânfora e depois por cardiazol. A primeira série de TC resultou na suspensão do estupor catatônico e na remissão parcial da psicose. Entretanto, a remissão foi breve e, a despeito de repetidas sessões de TC, ZL nunca esteve inteiramente livre de sintomas, nunca teve alta hospitalar e veio a falecer no Instituto em 1945. Na discussão do caso de ZL, tentamos explicar as possíveis razões das discrepâncias entre o relato de Meduna e as notas originais do prontuário médico.

Baran B, et al. / Rev Psiq Clín. 2008;35(4):165-9

Palavras-chave: Tratamento convulsivo, cânfora, cardiazol, László Meduna, história da psiquiatria.

\begin{abstract}
Convulsive therapy (COT) is a major European contribution to the psychiatric armamentarium and biological psychiatry. COT was introduced in psychiatry by László Meduna, a Hungarian neuropsychiatrist. All subsequent publications about the first patient treated with COT, Zoltán L (ZL), were based on Meduna's papers and autobiography. After 4 years of catatonic stupor, ZL received camphor-induced COT which resulted in full remission and discharge from the institution. The aim of this paper is to reconstruct ZL's case history from the original case notes-partly written by Meduna himself-which were recovered from the archives of the National Institute of Psychiatry and Neurology. The case notes show that ZL repeatedly received COT between 1934 and 1937, first with camphor and then with cardiazol induction. After the first course of COT the catatonic stupor was resolved and the psychotic symptoms subsided.
\end{abstract}

\footnotetext{
(Originalmente publicado como “The beginning of modern psychiatric treatment in Europe. Lessons from an early account of convulsive therapy”. Brigitta Baran, István Bitter, Gabor S. Ungvari, Zoltán Nagy and Gábor Gazdag. European Archives of Psychiatry and Clinical Neuroscience. 2008 May 26. [Epub ahead of print]. Com a gentil permissão de Springer Science+Business Media). [Tradução de Paulo C. Sallet, autorizada pelo editor]. 
However, the remission lasted for only a few months and was followed by a relapse. Despite repeated courses of COT, ZL never became symptom free again, was never discharged and died in the Institute in 1945 . This historical case is discussed from both the diagnostic and therapeutic points of view, and an attempt is made to explain the possible reasons for the discrepancies found between Meduna's account and ZL's case notes.

Baran B, et al. / Rev Psiq Clín. 2008;35(4):165-9

Key-words: Convulsive treatment, camphor, cardiazol, László Meduna, history of psychiatry.

\section{Introdução}

A partir da introdução da terapia por febre malárica para o tratamento da neurossífilis, em $1917^{1}$, nas duas décadas seguintes houve um esforço significativo na busca por terapias psiquiátricas: sonoterapia ${ }^{2}$, choque insulínico ${ }^{3}$, terapia convulsiva (TC) induzida por cânfora e, mais tarde, por cardiazol ${ }^{4}$, lobotomia frontal ${ }^{5}$ e uma versão modificada da TC, a eletroconvulsoterapia (ECT) ${ }^{6}$. A TC - cujos primórdios são o foco deste trabalho - é a única intervenção terapêutica remanescente na psiquiatria moderna, embora praticada de uma forma bastante aprimorada, como ECT?

Após experimentos com animais conduzidos em $1933^{8}$, a TC foi pela primeira vez tentada com pacientes pelo neuropsiquiatra húngaro László J. Meduna (18961964), em 1934, no Instituto Nacional Real de Neurologia e Psiquiatria, Budapeste, Hungria.

Meduna recebeu seu diploma de licenciatura em medicina em 1921, em Budapeste. Iniciou sua carreira científica como neuropatologista ${ }^{9,10}$, primeiro estudando a estrutura da glândula pineal e, mais tarde, as células da glia e a epilepsia. Em contraste com os achados cerebrais de pacientes epilépticos, que apresentavam profusa reação das células da glia, o cérebro de pacientes esquizofrênicos via de regra não apresentava gliose $\mathrm{e}^{11}$, sugerindo um antagonismo entre essas duas condições, aspecto apoiado também por dados clínicos ${ }^{12}$, o que levou Meduna à conclusão de que a esquizofrenia poderia ser tratada por meio de convulsões epilépticas. Meduna encontrou na cânfora a melhor substância para induzir convulsões epilépticas e realizou uma série de estudos em animais com esse composto ${ }^{8}$.

A apreciação retrospectiva da $\mathrm{TC}^{13} \mathrm{e}$ a autobiografia ${ }^{10}$ de Meduna, nas quais outros relatos se basearam ${ }^{7,14-19}$, identificaram o primeiro paciente tratado com TC como tendo sido Zoltán L (ZL), no qual foi injetada cânfora em óleo na manhã de 23 de janeiro de 1934. De acordo com o relato de Meduna, "este paciente vinha apresentando estupor catatônico há cerca de quatro anos. Ele não se movia, não comia, não cuidava de suas necessidades corporais e tinha de ser alimentado por sonda. [...] Ele recebeu cinco injeções de cânfora e, dois dias após a quinta injeção, na manhã de 10 de fevereiro, pela primeira vez em quatro anos saiu de seu leito, começou a falar, pediu o café da manhã [...] estava interessada em tudo o que o rodeava [...]" Meduna acompanhou o caso de ZL até deixar a Hungria, em 1939. De acordo com ele, a doença de ZL manteve-se em remissão até $1939^{10}$.

Uma recente pesquisa nos Arquivos do Instituto Nacional de Psiquiatria e Neurologia de Budapeste encontrou o registro clínico da primeira coorte dos pacientes de Meduna que receberam TC. Ao que tudo indica, Meduna foi traído pela memória quando escreveu sua análise ${ }^{13}$ e "autobiografia" ${ }^{10}$, cerca de 30 anos após a primeira TC.

Em uma perspectiva histórica mais ampla, é pouco relevante que Zoltán não tenha sido de fato o primeiro paciente a receber TC. Na verdade, dois outros pacientes catatônicos (JW e ER) tiveram o início de seus tratamentos em 2 de janeiro de 1934, tal como descrito no livro de Meduna, de 1937, sobre terapia convulsiva ${ }^{4}$. Mais importante do que a identificação do primeiro paciente é o curso da doença de ZL e sua resposta à TC, inicialmente induzida por cânfora e mais tarde pelo cardiazol (pentilenotetrazol, metrazol). No intuito de reavaliar o resultado da TC de ZL, reconstruímos aqui o curso de sua doença com base nos registros originais.

\section{Uma descrição da doença de ZL}

Aos 33 anos de idade, casado, eletricista, ZL foi admitido no Instituto Nacional Real Húngaro de Psiquiatria e Neurologia, Budapeste, em 18 de outubro de 1930. Essa foi sua primeira e única internação psiquiátrica.

Ele vinha apático e perturbado há algum tempo. O parecer médico encaminhando Zoltán para a instituição afirmava: "De acordo com o relato de sua família, nesta tarde ele brigou com a esposa de uma forma que os fez acreditar que a quisesse estrangular. [...] O paciente não lembra disso". O diagnóstico de admissão foi de "esquizofrenia".

Um dos irmãos de ZL e seu tio paterno apresentavam psicoses de natureza desconhecida. ZL contraiu sífilis em 1921, que foi "curada" possivelmente por meio da terapia por febre malárica*.

\footnotetext{
* Em 1917, o neuropsiquiatra austríaco Julius Wagner von Jauregg (1857-1940) descobriu que poderia inibir a progressão da demência paralítica (sífilis terciária) inoculando seus pacientes com o parasita da febre terçã benigna (Plasmodium malariae). Os pacientes apresentavam diversos episódios febris, algumas semanas mais tarde interrompidos pelo uso de quinina. A terapia por febre malárica mostrou-se benéfica na evolução da história natural da sífilis, teve seu uso rapidamente difundido na era pré-antibióticos, levando von Jauregg ao Prêmio Nobel em 1927. (NT)
} 
Seu pai, funcionário aposentado, era "um homem alto e tranqüilo"; sua mãe, "uma mulher alegre, obesa e de baixa estatura, que faleceu aos 53 anos de idade por problemas renais e cardíacos".

O desempenho escolar de ZL "era satisfatório; era uma criança muito vívida". Foi repetidamente reprovado no segundo ano do ensino médio e interrompeu os estudos. Tornou-se aprendiz de serralheiro, mas logo abandonou a função, porque se esquecia das coisas. Iniciou então uma formação como eletricista, mas não conseguiu obter as competências suficientes, tendo sido empregado apenas como assistente de eletricista. No final de 1918, fez o alistamento militar, mas não foi para o front. Em 1925, casou-se e teve uma filha saudável. Não há outras informações sobre sua esposa, exceto que ela ocasionalmente visitava o marido na instituição.

$\mathrm{Na}$ admissão, ZL referiu que as pessoas costumavam ser particularmente hostis para com ele, mas ultimamente elas se mostravam mais amigáveis e muitas vezes até mesmo desconhecidos acenavam para ele. Sentia que "as pessoas estavam passando coisas no seu rosto e os animais se esgueiravam diante de seus pés". Ele freqüentemente ouvia vozes estranhas, às vezes vindas de seu estômago, vozes cujo significado ele entendia apenas ocasionalmente. Achava que não era o pai de sua filha, alegando que seu irmão teria seduzido sua esposa. Não entendia por que estava sendo internado em uma instituição psiquiátrica, mas não se importava com isso por achar que ali fosse sua casa. Ele não sabia se estava realmente louco.

Antes do aparecimento da doença "[...] apresentava sintomas neurastênicos, cansaço, desinteresse, apatia e pensamento confuso. Às vezes sentia seu corpo terrivelmente quente; noutras vezes, muito frio. Quando examinado não respondia ao ser questionado, mas continuou falando com a esposa sobre sua filha. Sentava-se olhando para o nada e dizia que estava ouvindo vozes de dentro de seu corpo [...] Há cerca de quatro semanas havia pedido que a esposa lhe desse veneno para se matar".

Durante a admissão "estava calmo, quase mudo e só respondia às perguntas com monossílabos”. Apresentava uma "constituição astênica", com 1,61 m de altura e $51 \mathrm{~kg}$ de peso. Os sinais vitais e o exame neurológico estavam dentro de limites fisiológicos. O exame do estado mental enfatizava prejuízo na vigilância e déficit de atenção, desorientação no tempo, baixa capacidade intelectual, comportamento esquivo e hesitante, além de alucinações visuais e auditivas.

Entre 20 e 28 de outubro de 1930, ZL estava "isolado" e "deprimido", "não estava interessado no ambiente" e "ficava deitado sob o cobertor o dia todo", além de estar "afetado por delírios".

No $12^{\circ}$ dia de internação, uma avaliação psiquiátrica concluiu que "[...] Durante toda a entrevista, o indivíduo observado ficou sentado em sua cadeira com a cabeça abaixada, não respondeu às perguntas, no máximo movia os lábios sem emitir som; além disso, mostrou-se completamente inabordável. De acordo com os registros, o paciente batia a cabeça na parede e teria tentado estrangular a si próprio. Ele sofre de uma psicose esquizofrênica, o que justifica seu tratamento em um hospital psiquiátrico".

De novembro de 1930 até 18 de abril de 1931, ZL mostrou-se principalmente negativista, mudo, sem interesse no ambiente e se alimentou muito pouco. Costumava ficar deitado em sua cama ou em pé de frente para a parede atrás da porta o dia todo. Sua condição flutuava ligeiramente: em raras ocasiões foi capaz de passar uma semana em casa ou caminhava no parque do Instituto com sua esposa. Seu peso caiu para $48 \mathrm{~kg}$; por conseguinte, em 18 de abril de 1931 foi iniciada alimentação por sonda nasogástrica, que continuou sendo necessária até o início de 1934, quando sua TC teve início. Os anos de 1932 e 1933 se passaram sem quaisquer alterações significativas na sua condição.

Após sua admissão, ZL foi tratado com ópio e estricnina, sem maiores efeitos.

\section{Descrição da terapia convulsiva de ZL}

Em 23 de janeiro de 1934, ZL começou a receber TC induzida por cânfora e cardiazol, cujo progresso é aqui sumariamente resumido. Entre 23 de janeiro e 17 de fevereiro, ZL recebeu oito sessões de TC com $4 \mathrm{mg}$ a $5 \mathrm{mg}$ de cânfora ministrada por via intramuscular, resultando em sete convulsões com duração entre 30 e 60 segundos. O efeito imediato, embora de curta duração, foi nada menos do que surpreendente: o paciente antes em mutismo, alimentado por sonda, "levantou-se sozinho, conversou com sagacidade, perguntou sobre sua esposa”. Dentro de uma semana, entretanto, ZL voltou a ficar mudo e recluso, mas seguindo-se à $9^{\mathrm{a}}$ sessão de TC (8 de março) houve uma longa remissão durante dois meses seguidos: "ele falou com inteligência [...] de modo expressivo, alegre, comunicativo e educado. [...] faz reparos na oficina de trabalho [...] sai para a cidade duas vezes por semana". De 12 de julho até 28 de agosto, ZL recebeu outras sete sessões de TC com sete episódios convulsivos com duração de 50 a 70 segundos; as últimas quatro sessões foram induzidas por $40 \mathrm{cg}$ de cardiazol por via intravenosa: "Ele solicitou alimentos [...] pediu que lhe dessem alguma ocupação, que agora tem levado a cabo de forma diligente. De modo geral, uma excelente remissão". Essa foi a segunda remissão parcial, que também foi curta. De 18 de outubro de 1934 até 2 de abril de 1935, ZL recebeu duas TC induzidas por cânfora e 12 TC induzidas por cardiazol, sete das quais resultaram em convulsões. ZL ficou livre de alucinações e delírios, permaneceu "calmo e alegre" e, aparte o comportamento algo desajeitado, "ele encontra-se em remissão [...] trabalha no jardim”. Em 14 de agosto do mesmo ano ele encontrava-se novamente catatônico e ostentosamente psicótico; a despeito de outras seis sessões de TC induzidas por cardiazol, sua condição piorou 
progressivamente ao longo de 1935 . Não foram realizadas sessões de TC em 1936 e houve apenas três sessões induzidas por cardiazol em 1937, das quais apenas uma resultou em uma convulsão de 45 segundos. A partir de então não foram mais ministradas sessões de TC.

\section{O curso da doença de ZL após 1938 até a sua morte}

De 1938 a 1939 ZL permaneceu em mutismo. Por vezes mostrava-se confuso e não demonstrava interesse em seu ambiente; comia, dormia bem, mas não mais trabalhou. Entre 22 de outubro e 15 de novembro de 1940, ele recebeu insulina em 15 ocasiões. Foram induzidos cinco comas e três crises epilépticas, sem qualquer efeito clínico. Em 1941, "ele exibe maneirismos e mostra-se tímido, embora calmo e tranqüilo. O seu humor varia, às vezes mostra-se deprimido durante dias, caminhando a esmo pelo corredor, sem responder, apenas sorrindo; noutras vezes, está mais comunicativo, brincalhão". Em 1942, "às vezes mostra-se irritável, proclama seus delírios, mas permanece bem com os outros pacientes; mostra-se calmo, mudo, isolado, sem mostrar interesse pelo ambiente, embora ocasionalmente torne-se ansioso, irritável e nervoso". Em 1943-1944, mais e mais freqüentemente, “ [...] perturba os outros pacientes, [...] é desleixado, negligencia cuidados com a aparência, [...] rasga suas roupas e só as usa dessa forma, [...] deita-se no chão [...]". Com regularidade envolve-se em brigas com outros pacientes. Continua apresentando alucinações e delírios paranóides. Desde o início de 1945, “[...] mostra-se enfraquecido, em declínio físico, deitado na cama, pálido, não tem febre, mostra-se perturbado, não demonstra interesse no ambiente, embora tome suas refeições e durma à noite". Em 23 de fevereiro de 1945, "na cama, quieto, confuso, não aceitou café da manhã. Sem febre. Respira com dificuldade de manhã. Morre de dementia praecox às $12 \mathrm{~h} 45$ ”.

\section{Discussão}

Seguindo-se à TC, a condição de ZL apresentou um progressivo deterioro ao longo de mais de três anos, caracterizada por negativismo, estupor, delírios pobremente estruturados e alucinações auditivas. Os registros revelam que a avaliação de Meduna sobre a resposta de ZL à TC foi demasiado otimista. Entre 1934 e 1935, ZL recebeu um total de 36 sessões de TC distribuídas em quatro séries e resultando em dois períodos de remissão parcial, cada um dos quais com dois a três meses de duração. De modo geral, não houve qualquer melhora duradoura no seu estado mental, que não a supressão do estupor e da recusa de alimentos.

Em janeiro de 1937, foram ministradas outras três TC com baixas doses de cardiazol, que produziram apenas uma convulsão sem benefício terapêutico. A condição tampouco melhorou com as 15 sessões induzidas por insulina ministradas em 1940, resultando em cinco comas e três crises epilépticas. Entre 1940 e 1945, houve novo declínio gradual em ambas as condições física e mental, embora a alimentação por sonda não tenha mais sido necessária e nem tenha se desenvolvido estupor. ZL nunca teve alta da instituição após sua admissão em 1930 .

A causa da morte do paciente permanece incerta. Não há informações de autopsia disponíveis. Sua morte pode ser explicada pela desnutrição e pelas más condições de higiene devidas à inatividade. $\mathrm{Em} 1^{\circ}$ de janeiro de 1945 seu peso era de apenas $43 \mathrm{~kg}$.

O diagnóstico de esquizofrenia de ZL, estabelecido por eminentes psiquiatras treinados no espírito da psiquiatria clássica alemã, nunca foi questionado. O caráter quase contínuo, o predomínio de alucinações auditivas humor-incongruentes, a sempre presente suspicácia e ideações paranóides vagas, o isolamento social extremo, o comportamento idiossincrático e bizarro, juntamente com o deterioro progressivo na evolução da doença tornam o diagnóstico de esquizofrenia bastante consistente.

Que lições podem ser tiradas do caso de ZL? A falta de remissão mais prolongada na doença do paciente pode ser devida ao relativamente pequeno número de sessões de TC; atualmente alguns autores afirmam que na esquizofrenia podem ser necessárias cerca de 20 sessões de ECT para que haja remissão mais persistente ${ }^{20,21}$. Desde que teve início a TC, Zoltán nunca mais ficou gravemente estuporoso nem necessitou ser alimentado por sonda. Há um amplo consenso na psiquiatria moderna de que a terapia convulsiva é o tratamento mais eficaz para a catatonia e o estupor de variadas origens ${ }^{22}$.

Em uma perspectiva mais ampla, o caso de ZL é um exemplo do entusiasmo que ocorre sempre que uma nova modalidade de tratamento é introduzida. O enorme entusiasmo inicial será em breve temperado por uma avaliação mais crítica, quando o tratamento, com as limitações inerentes, finalmente encontra seu lugar no arsenal terapêutico. A aceitação de um novo tratamento depende de forças éticas e socioculturais ("garantias éticas e sociais”"23.

Em razão de um controle mais rigoroso da sociedade sobre a profissão psiquiátrica nos últimos 20 a 30 anos e de maior preocupação com segurança clínica, as indicações, a eficácia e o risco-benefício dos novos tratamentos são identificados e as providências cabíveis, tomadas com muito mais presteza do que antes.

O lugar de Meduna na história da psiquiatria como um dos pioneiros da psiquiatria é inquestionável. Meduna introduziu um novo tratamento psiquiátrico baseado nos seus estudos neuropatológicos e observações clínicas anteriores. Como sugerido por Fink ${ }^{24}$, a introdução da TC na psiquiatria foi uma consequiência lógica da atividade científica anterior de Meduna e não uma descoberta ocasional. A terapia pela febre malárica

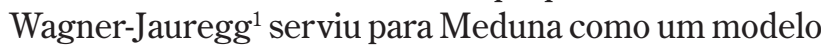
de cura de uma doença mediante a indução de outro 
estado patológico. A TC inaugurou uma mudança de paradigma na abordagem do conceito de esquizofrenia, na medida em que desafiou com sucesso o niilismo terapêutico em que se encontravam as psicoses endógenas. A introdução da TC deu início à investigação biológica em psiquiatria e também impulsionou o ânimo e a autoestima profissional dos psiquiatras ${ }^{19}$.

De um ponto de vista prático, a TC representou um verdadeiro avanço na medida em que se mostrou muito mais eficaz do que qualquer intervenção terapêutica até então utilizada. A TC rapidamente se espalhou por todo o mundo; em 1938, foi introduzida em 92 instituições psiquiátricas na Inglaterra e no País de Gales, com 3.531 doentes tendo sido tratados com TC ${ }^{25}$. Até 1940 tinham sido publicados 3.900 artigos sobre TC, incluindo-se a eletroconvulsoterapia, o que ilustra a excitação que o novo método terapêutico gerou na comunidade científica $^{13}$. Mais importante ainda, ao longo das últimas sete décadas, a TC vem reduzindo o sofrimento de dezenas de milhares de pacientes.

\section{Referências}

1. Whitrow M. Julius Wagner-Jauregg (1857-1940). London: SmithGordon; 1993.

2. Klaesi J. Über die therapeutische Anwendung der "Dauernarkose" mittels Somnifen bei Schizophrenen. Z Ges Neurol Psychiat. 1922;74:557.

3. Sakel M. Neue Behandlung der Morphinsucht. (Eine Insulinkur beseitigt die Abstinentenzerscheinungen durch Ausgleich des während der Entziehung gestörten Gleichgewichtes im vegetativen Nervensystem) (in German). Zschr Neurol Psychiat. 1933;143:506-34.

4. Meduna L. Die Konvulsionstherapie der Schizophrenie. Halle: Carl Marhold Verlagsbuchhandlung; 1937.

5. Berrios GE. The scientific origins of electroconvulsive therapy: a conceptual history. Hist Psychiatry. 1997;8:105-19.
6. Passione R. Italian psychiatry in an international context: Ugo Cerletti and the case of electroshock. Hist Psychiatry. 2004;15:83-104.

7. Fink M. Induced seizures as psychiatric therapy. J ECT. 2004;20:133-6.

8. Meduna LJ. Über experimentelle Campherepilepsie. Arch Psychiat Nervenkr. 1934;102:333-9.

9. Baran B, Bitter I, Fink M, Gazdag G, Shorter E. Károly Schaffer and his school: the birth of biological psychiatry in Hungary, 1890-1940. Eur Psychiatry 2007 Dec 8; [Epub ahead of print].

10. Meduna. Autobiography. Convulsive Ther. 1985;1:43-57, 121-38.

11. Hechst B. Zur Histopathologie der Schizophrenie mit besonderer Berücksichtigung der Ausbreitung des Prozesses. Z Ges Neurol Psychiatr. 1931;134:163-267.

12. Nyír G, Jablonszky A. Some data concerning the prognosis of epilepsy, with special attention to constitution. (In Hungarian). Orv Hetil. 1929;28:679-81.

13. Meduna L. The convulsive treatment: a reappraisal. J Clin Exp Psychopathol. 1954;15:219-33.

14. Berrios GE. The origins of psychosurgery: Shaw, Burckhardt and Moniz. Hist Psychiatry. 1997;8:61-81.

15. Fink M. Convulsive therapy: a review of the first 55 years. J Affective Disorders. 2001;63:1-15.

16. Fink M. Meduna and the origins of convulsive therapy. Am J Psychiatry. 1984;141:1034-41.

17. Fink M, Taylor MA. Catatonia: A Clinician's Guide to Diagnosis and Treatment. United Kingdom: Cambridge University Press, Cambridge, 2003.

18. Kalinowsky LB. History of convulsive therapy. Ann N Y Acad Sci. 1986;462:1-4.

19. Shorter E, Healy D. Shock therapy: the history of electroconvulsive treatment in mental illness. New Brunswick: Rutgers University Press, 2007.

20. Chanpattana W, Andrade C. ECT for treatment-resistant schizophrenia: a response from the Far East to the UK NICE report. J ECT. 2006;22:4-12.

21. Tharyan $\mathrm{P}$, Adams CE. Electroconvulsive therapy in schizophrenia. Cochrane Database System Review. 2002(2):CD000076.

22. Fink M, Taylor, MA. The many varieties of catatonia. Eur Arch Clin Neurosci. 2001;251(Suppl. 1):8-13.

23. Kalinowsky LB. The discoveries of somatic treatments in psychiatry: facts and myths. Compr Psychiatry. 1980;21:428-35.

24. Fink M. ECT: Serendipity of logical outcome? Psychiatric Times. 2004;21(1).

25. Board of Control for England \& Wales. The Twenty-fifth Annual report of the Board of Control, 1938. London: HMSO; 1939. 